\title{
Eikonal Equations for Null Radial Geodesics in the Schwarzschild Metric
}

\author{
Miguel Socolovsky \\ Instituto de Ciencias Nucleares, Universidad Nacional Autónoma de México, Cd. Universitaria, 04510, Ciudad de \\ México, México \\ Email: socolovs@nucleares . unam.mx
}

\begin{abstract}
We study the eikonal function $\phi$ corresponding to outgoing and ingoing radial null geodesics (light rays in the short wave length limit) in the Schwarzschild spacetime. Contrary to the behavior of the expansion scalar $\Theta$ at the singularities (past and future), $\phi$ turns out to be finite at $r=0$ (except for light travelling along the horizons) and inversely proportional to $M$, the mass of the black hole, and so proportional to the Hawking temperature.
\end{abstract}

Keywords: Schwarzschild spacetime, eikonal function, radial light rays

\section{Introduction}

In the short wave length limit, electromagnetic radiation (photons) is represented by light rays (geometric optics) [1] which, in turn, in the context of general relativity, are represented by null geodesics. These, in particular, are crucial tools for exploring the structure of black holes, both in the neighbourhood of their horizons or their singularities. On the other hand, the behavior of light rays in the geometric optics approximation is governed by the eikonal function $\phi[2]$, whose gradients $\partial_{\mu} \phi$ give the wave vectors $\tilde{k}^{\mu}$ of the light propagation which, in turn, are proportional to the tangent vectors $k^{\mu}$ of the corresponding geodesics. By dimensional reasons, the proportionality constant has units of length, which for the Schwarzschild metric is the mass of the black hole (1/2 of the Schwarzschild radius). $\phi$ satisfies a wave equation which reminds the ondulatory nature of light; beyond that, it can be quantized, leading to the photon concept [1].

In the absence of a theory of quantum gravity, any treatment of radiation propagation in black holes must be semiclassical. In this article, strictly remaining at a classical level, we ask for the behavior of the eikonal function associated with the simplest null geodesic congruences in the Schwarzschild-KruskalSzekeres [3]-[4] spacetime: namely, the radial ones. The main interest lies in the behavior of $\phi$ near the singularities of the ideal black/white eternal hole (section 5). The past singularity acts as a source of outgoing light rays which travel towards the left and right future null infinities, while the left and right past null infinities can be considered sources of incoming light rays ending in the future singularity. In their paths, the light rays cross the future and the past horizons, giving us information of $\phi$ at these points. For comparison, we review the behavior at the singularities and horizons of the expansion scalars of the Raychaudhuri equation [5]-[6] obeyed by the above mentioned null geodesic congruences [7] (section 4). The description of the radial null geodesics in Eddington-Finkelstein [8] and Kruskal-Szekeres-Penrose [9]-[10] coordinates is given respectively in sections 2 and 3, while section 6 is reserved to a final discussion.

Note. We use metrics with signature $(+,-,-,-)$, and the geometrical system of units $G=c=1$.

\section{Radial Null Geodesics in E-F Coordinates}

\section{$2.1 \quad$ Geodesics}

The Schwarzschild metric $d s_{S c h w}^{2}$ for radial null geodesics (light rays) is given by

$$
g_{t t} d t^{2}+g_{r r} d r^{2}=f d t^{2}-f^{-1} d r^{2}=0
$$


where

$$
f=1-2 M / r \text {. }
$$

The angular part of the metric is $\left(g_{\theta \theta}, g_{\varphi \varphi}\right)=\left(-r^{2},-r^{2} \sin ^{2} \theta\right)$, while for the inverse metric one has $\left(g^{t t}, g^{r r}, g^{\theta \theta}, g^{\varphi \varphi}\right)=\left(f^{-1},-f,-r^{-2},-r^{-2} \sin ^{-2} \theta\right)$.

In terms of the radial ("tortoise") coordinate

$$
\tilde{r}(r)=r+2 M \ln \left|\frac{r}{2 M}-1\right|
$$

the solution of (1) is

$$
t= \pm \tilde{r}(r)+\text { const. }
$$

$\tilde{r} \in(-\infty,+\infty)$ with $\tilde{r} \rightarrow+\infty$ for $r \rightarrow+\infty$ and $\tilde{r} \rightarrow-\infty$ for $r \rightarrow(2 M)_{+}$. In the $(t, \tilde{r})$ plane, the upper (lower) sign represents outgoing (ingoing) light rays, respectively $\frac{d t}{d \tilde{r}}= \pm 1$. In these coordinates the light cones (L.C.'s) are the same (at $\left.45^{\circ} / 135^{\circ}\right)$ for all $(t, \tilde{r})$ i.e. they do not bend (Figure $(1 \alpha)$ ); however, $(t, \tilde{r})$ are not "good" coordinates since $\tilde{r} \rightarrow-\infty$ at the horizon $2 M$. Instead, in coordinates $(t, r)$, L.C.'s bend as shown in Figure $(1 \beta)$. In terms of the Eddington-Finkelstein (E-F) coordinates

$$
u:=t-\tilde{r}
$$

and

$$
v:=t+\tilde{r}
$$

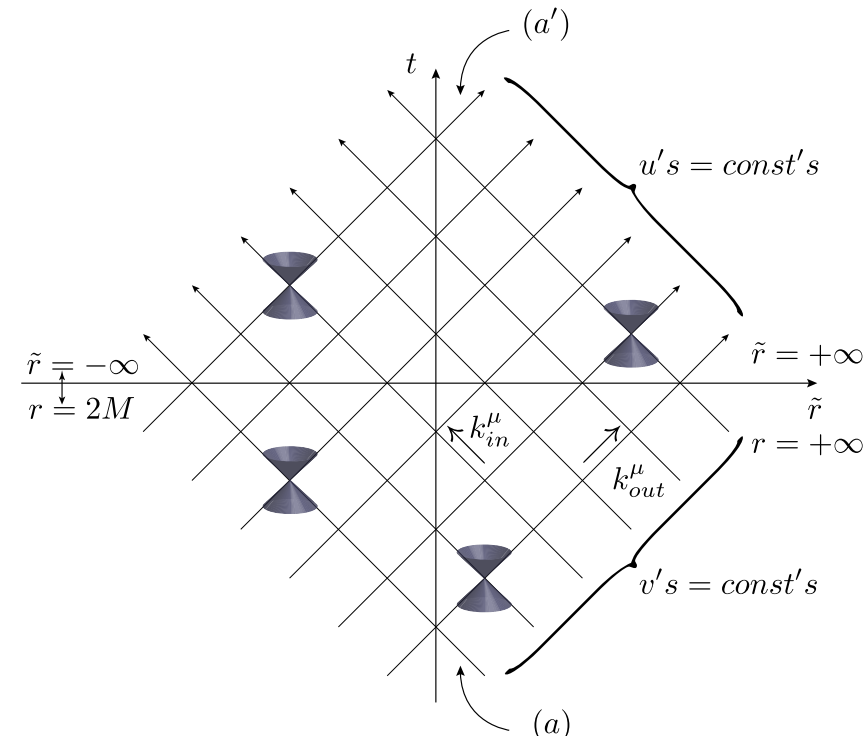

$(\alpha)$

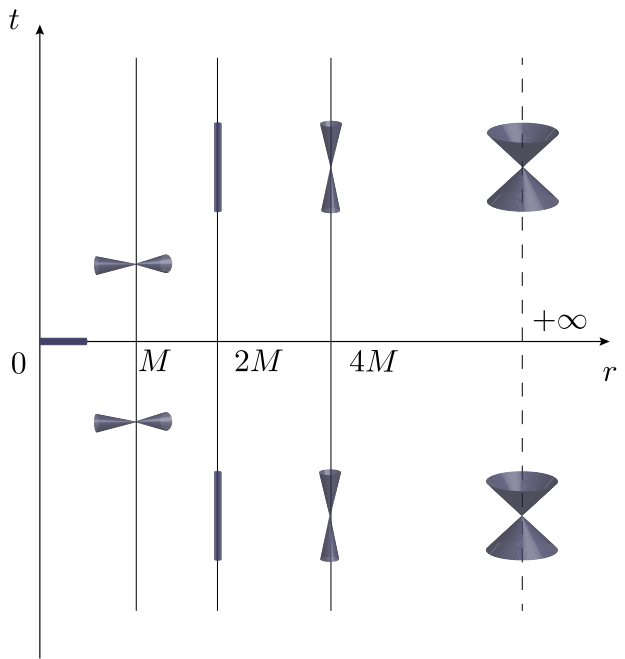

$(\beta)$

Figure 1. $(\alpha)$ : L.C.'s in $(t, \tilde{r})$ coordinates, $(\beta)$ : L.C's in $(t, r)$ coordinates

$(u, v \in(-\infty,+\infty)), \frac{d t}{d \tilde{r}}= \pm 1$ respectively correspond to $u^{\prime} s=$ const.'s, ((a) rays) and $v^{\prime} s=$ const.'s, ((a) rays). In Figure $(1 \alpha)$ are also indicated the corresponding tangent vectors $k_{i n}^{\mu}$ and $k_{\text {out }}^{\mu}$ (see below for its definition).

If we define the temporal coordinates $\tilde{t}$ and $\bar{t}$ through

$$
\tilde{t}:=t-2 M \ln \left|\frac{r}{2 M}-1\right|, \quad \bar{t}:=t+2 M \ln \left|\frac{r}{2 M}-1\right|
$$

we obtain

$$
u=t-r-2 M \ln \left|\frac{r}{2 M}-1\right|=\tilde{t}-r
$$


and

$$
v=t+r+2 M \ln \left|\frac{r}{2 M}-1\right|=\bar{t}+r .
$$

In (8), $\tilde{t}<\infty$ at $r=2 M$ requires $t=-\infty$ (past horizon $h_{-}$), and in (9), $\bar{t}<\infty$ at $r=2 M$ requires $t=+\infty$ (future horizon $h_{+}$). The pairs $(v, r)$ and $(u, r)$ together with the angular coordinates $(\theta, \varphi)$, respectively define the E-F advanced and retarded spacetimes $E F A$ and $E F R$, with metrics

$$
d s_{E F A}^{2}=f d v^{2}-2 d v d r-r^{2} d \Omega_{2}^{2}
$$

and

$$
d s_{E F R}^{2}=f d u^{2}+2 d u d r-r^{2} d \Omega_{2}^{2}
$$

with $d \Omega_{2}^{2}=d \theta^{2}+\sin ^{2} \theta d \varphi^{2}$. Since both $d s_{E F A}^{2}$ and $d s_{E F R}^{2}$ are regular at $r=2 M$, they are extensions of the Schwarzschild metric beyond the horizon. In the $E F A$ case, $0<r<2 M$ is the black hole region $(I I)$, while in the $E F R$ case, $0<r<2 M$ is the white hole region $\left(I I^{\prime}\right)$. In both cases, $r>2 M$ is called region $(I)$ or $\mathcal{U}$.

From (10) and (11) radial light rays obey, respectively,

$$
(f d v-2 d r) d v=0
$$

and

$$
(f d u+2 d r) d u=0 .
$$

In $(12), d v=0$ i.e. $v=$ const., are the ingoing $(a)$ rays in Figure $(1 \alpha)$, while the solutions of $f d v-2 d r=0$ are the outgoing light rays $(c)$ in region $(I)$ and the ingoing light rays $(b)$ in region $(I I)$, Figure $(2 \alpha)$.

In (13), $d u=0$ i.e. $u=$ const., are the outgoing $\left(a^{\prime}\right)$ rays in Figure $(1 \alpha)$, while the solutions of $f d u+2 d r=0$ are the ingoing light rays $\left(c^{\prime}\right)$ in region $(I)$ and the outgoing light rays $\left(b^{\prime}\right)$ in region $\left(I I^{\prime}\right)$, Figure $(2 \beta)$.

So, in the E-F extension of the Schwarzschild metric, there are six radial future directed null geodesic congruences: three ingoing $\left((a),(b),\left(c^{\prime}\right)\right)$ and three outgoing $\left(\left(a^{\prime}\right),\left(b^{\prime}\right),(c)\right)$. In the unified view of the Kruskal-Szekeres spacetime, the number of independent set of radial null geodesic congruences reduces to four: $\left(a^{\prime}\right) \equiv(c),\left(c^{\prime}\right) \equiv(a),(b)$, and $\left(b^{\prime}\right)$. (Given an open set $U$ of the spacetime, a congruence of (massless or massive) geodesics in $U$ is a family or set of curves in $U$ which obey the geodesic equation, and such that through each point $p$ in $U$ passes one and only one curve from the family.)

\subsection{Geodesic Equations}

If $\lambda$ is an affine parameter for a geodesic, the tangent vector to the curve

$$
k^{\mu}=\frac{\partial x^{\mu}}{\partial \lambda}, \quad k^{2}=g_{\mu \nu} k^{\mu} k^{\nu}
$$

with $k^{2}=0$ for a null geodesic and $\left[k^{\mu}\right]=[L][\lambda]^{-1}$, obeys the geodesic equation

$$
\begin{aligned}
k \cdot D\left(k^{\alpha}\right)=k^{\mu} D_{\mu}\left(k^{\alpha}\right)=k^{\mu} k_{; \mu}^{\alpha} & =k^{\mu}\left(k_{, \mu}^{\alpha}+\Gamma_{\mu \nu}^{\alpha} k^{\nu}\right) \\
& =k_{, \mu}^{\alpha} k^{\mu}+\Gamma_{\mu \nu}^{\alpha} k^{\mu} k^{\nu}=\frac{d}{d \lambda} k^{\alpha}+\Gamma_{\mu \nu}^{\alpha} k^{\mu} k^{\nu}=0
\end{aligned}
$$

where $\Gamma_{\mu \nu}^{\alpha}=\Gamma_{\nu \mu}^{\alpha}$ are the Christoffel symbols associated with the Schwarzschild metric:

$$
\begin{aligned}
\Gamma_{t r}^{t}=\frac{M}{r^{2} f}, \Gamma_{t t}^{r}=\frac{M}{r^{2}} f, \Gamma_{r r}^{r} & =-\frac{M}{r^{2} f}, \Gamma_{\theta \theta}^{r}=-r f, \Gamma_{\varphi \varphi}^{r}=-r f \sin ^{2} \theta, \Gamma_{r \theta}^{\theta} \\
& =\frac{1}{r}, \Gamma_{\varphi \varphi}^{\theta}=-\sin \theta \cos \theta, \Gamma_{r \varphi}^{\varphi}=\frac{1}{r}, \Gamma_{\theta \varphi}^{\varphi}=\operatorname{cotg} \theta .
\end{aligned}
$$

(All other symbols vanish.) 
For the outgoing geodesics $\left(a^{\prime}\right), u=$ const.,

$$
k_{\mu}^{\text {out }}=\partial_{\mu} u=\left(\partial_{t} u, \partial_{r} u\right)=\left(k_{t}^{\text {out }}, k_{r}^{\text {out }}\right)=\left(1,-f^{-1}\right),
$$

while for the ingoing geodesics $(a), v=$ const.,

$$
k_{\mu}^{i n}=\partial_{\mu} v=\left(\partial_{t} v, \partial_{r} v\right)=\left(k_{t}^{i n}, k_{r}^{i n}\right)=\left(1, f^{-1}\right),
$$

with

$$
k_{\text {out }}^{\mu}=g^{\mu \nu} k_{\nu}^{\text {out }}=\left(k_{\text {out }}^{t}, k_{\text {out }}^{r}\right)=\left(f^{-1}, 1\right)
$$

and

$$
k_{i n}^{\mu}=g^{\mu \nu} k_{\nu}^{i n}=\left(k_{i n}^{t}, k_{i n}^{r}\right)=\left(f^{-1},-1\right) .
$$

A straightforward calculation shows that $k_{\text {out }}^{\mu}$ and $k_{i n}^{\mu}$ obey the geodesic equations

$$
\frac{d}{d r} k_{\text {out }}^{\mu}+\Gamma_{\nu \rho}^{\mu} k_{\text {out }}^{\nu} k_{\text {out }}^{\rho}=0
$$

and

$$
\frac{d}{d(-r)} k_{i n}^{\mu}+\Gamma_{\nu \rho}^{\mu} k_{i n}^{\nu} k_{i n}^{\rho}=0
$$

So, $u=$ const. geodesics have affine parameter $\lambda_{\text {out }}=r$, while $v=$ const. geodesics have affine parameter $\lambda_{\text {in }}=-r$, with $k_{\text {out }}^{\mu}$ and $k_{\text {in }}^{\mu}$ dimensionless i.e. $\left[k_{\text {out }}^{\mu}\right]=\left[k_{i n}^{\mu}\right]=[L]^{0}$.

For the $(b),(c),\left(b^{\prime}\right)$ and $\left(c^{\prime}\right)$ geodesics we use $t=t(r)$ respectively given by $r+2 M \ln \left(1-\frac{r}{2 M}\right)+$ const., $r+2 M \ln \left(\frac{r}{2 M}-1\right)+$ const., $-r-2 M \ln \left(1-\frac{r}{2 M}\right)+$ const., and $-r-2 M \ln \left(\frac{r}{2 M}-1\right)+$ const., which can be derived from (5)-(9), obtaining

$$
\left.k_{\text {in }}^{\mu}\right|_{(b)}=\left(-f^{-1},-1\right),\left.k_{\text {out }}^{\mu}\right|_{(c)}=\left(f^{-1}, 1\right),\left.k_{\text {out }}^{\mu}\right|_{\left(b^{\prime}\right)}=\left(-f^{-1}, 1\right),\left.k_{\text {in }}^{\mu}\right|_{\left(c^{\prime}\right)}=\left(f^{-1},-1\right)
$$

and geodesic equations (21) and (22) respectively for outgoing and ingoing rays, with $r$ and $-r$ the corresponding affine parameters. These results can be summarized in the equations

$$
\frac{d}{d r} k^{t} \pm \frac{2 M}{f r^{2}} k^{t} k^{r}=0
$$

and

$$
\frac{d}{d r} k^{r} \pm \frac{M}{r^{2}}\left(f\left(k^{t}\right)^{2}-f^{-1}\left(k^{r}\right)^{2}\right)=\frac{d}{d r} k^{r}=0
$$

with the upper (lower) sign corresponding to outgoing (ingoing) geodesics, and $f\left(k^{t}\right)^{2}-f^{-1}\left(k^{r}\right)^{2}=k^{2}=0$.

\section{Kruskal-Szekeres-Penrose Picture}

The use of the Kruskal-Szekeres coordinates $V$ and $U$ allows the maximal extension of the Schwarzschild metric, leading to the appearance of the new region $\left(\left(I^{\prime}\right) \equiv \overline{\mathcal{U}}\right)$ with $r>2 M$ and $U<0$. (In Figures $3 \alpha$ and $3 \beta$ we show the corresponding Penrose diagrams with coordinates $\tau \in[-\pi / 2,+\pi / 2], \rho \in[-\pi,+\pi]$, with $V+U=\operatorname{tg}\left(\frac{\tau+\rho}{2}\right), V-U=\operatorname{tg}\left(\frac{\tau-\rho}{2}\right)$.) In each region, the dependence of $U$ and $V$ with $t$ and $r$ is given as follows:

Region $(I) \equiv \mathcal{U}, r>2 M, U>0$ :

$$
\begin{aligned}
& V(t, r)=\sqrt{r / 2 M-1} e^{r / 4 M} S h(t / 4 M), \\
& U(t, r)=\sqrt{r / 2 M-1} e^{r / 4 M} C h(t / 4 M) .
\end{aligned}
$$

Region $(I I) \equiv B H, r<2 M, V>0$ :

$$
V(t, r)=\sqrt{1-r / 2 M} e^{r / 4 M} C h(t / 4 M),
$$




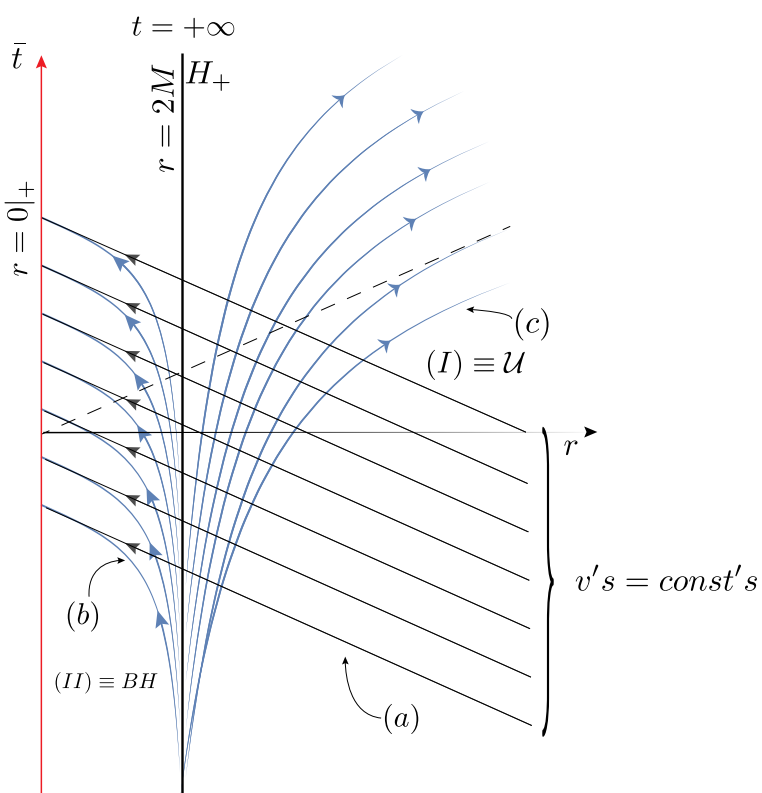

$(\alpha)$

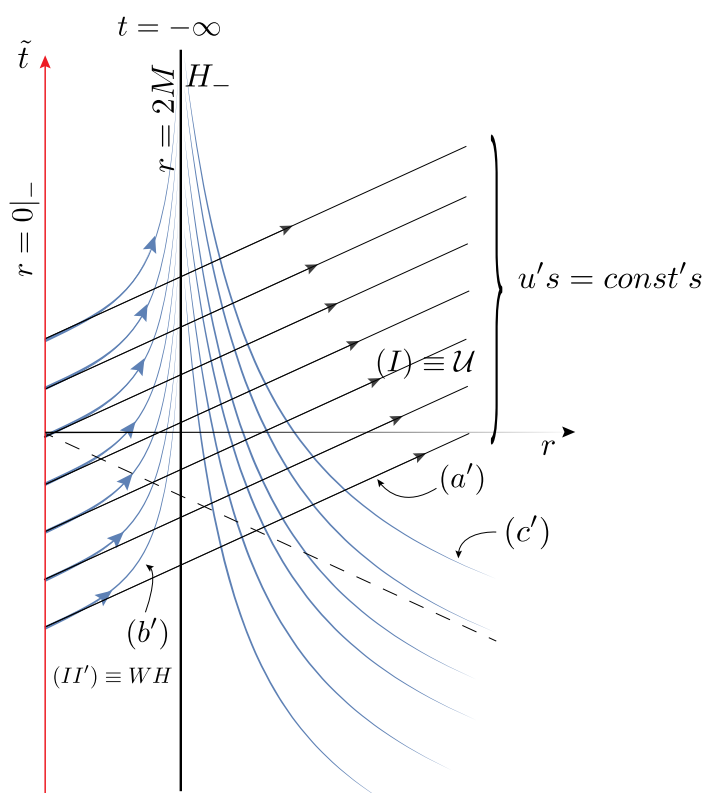

$(\beta)$

Figure 2. Radial null ingoing and outgoing geodesics in $B H$ and $\mathcal{U}(\alpha)$ and $W H$ and $\mathcal{U}(\beta)$

$$
U(t, r)=\sqrt{1-r / 2 M} e^{r / 4 M} S h(t / 4 M) .
$$

Region $\left(I I^{\prime}\right) \equiv W H, r<2 M, V<0$ :

$$
\begin{aligned}
& V(t, r)=-\sqrt{1-r / 2 M} e^{r / 4 M} C h(t / 4 M), \\
& U(t, r)=-\sqrt{1-r / 2 M} e^{r / 4 M} S h(t / 4 M) .
\end{aligned}
$$

Region $\left(I^{\prime} \equiv \overline{\mathcal{U}}\right), r>2 M, U<0$ :

$$
\begin{aligned}
& V(t, r)=-\sqrt{r / 2 M-1} e^{r / 4 M} S h(t / 4 M), \\
& U(t, r)=-\sqrt{r / 2 M-1} e^{r / 4 M} C h(t / 4 M) .
\end{aligned}
$$

All radial null geodesics become straight lines at $45^{\circ} / 135^{\circ}$ and, using the dependence $t=t(r)$, one obtains the picture shown in Figures $3 \alpha$ and $3 \beta$ :

geodesics $\left(a^{\prime}\right)$ : past singularity at $r=0 \rightarrow$ right future null infinity $J_{R}^{+}$,

geodesics $(a)$ : right past null infinity $J_{R}^{-} \rightarrow$ future singularity at $r=0$,

geodesics $\left(b^{\prime}\right)$ : past singularity at $r=0 \rightarrow$ left future null infinity $J_{L}^{+}$,

geodesics $(b)$ : left past null infinity $J_{L}^{-} \rightarrow$ future singularity at $r=0$,

geodesics $(c) \equiv$ geodesics $\left(a^{\prime}\right)$ beyond $r=2 M$,

geodesics $\left(c^{\prime}\right) \equiv$ geodesics $(a)$ up to $r=2 M$.

\section{Raychaudhuri Equations and Expansion Coefficients}

As is well known, any congruence of affine parametrized null geodesics in a spacetime obey the Raychaudhuri equation

$$
\frac{d \Theta}{d \lambda}=-\frac{1}{2} \Theta^{2}+\omega_{\mu \nu} \omega^{\mu \nu}-\sigma_{\mu \nu} \sigma^{\mu \nu}-R_{\mu \nu} k^{\mu} k^{\nu}
$$

where: 


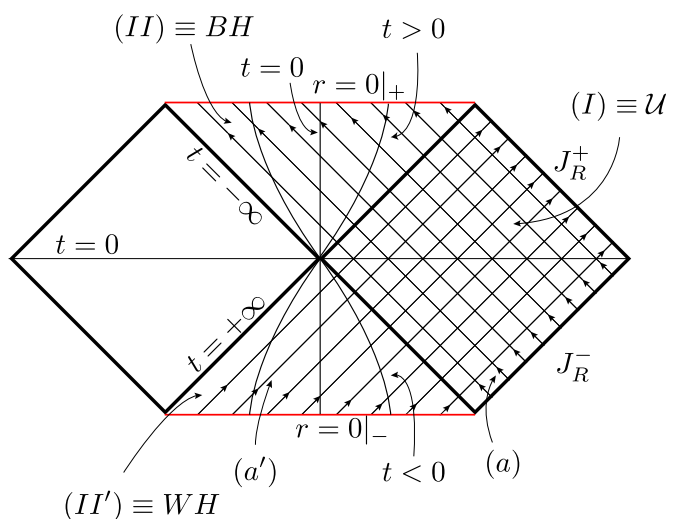

$(\alpha)$

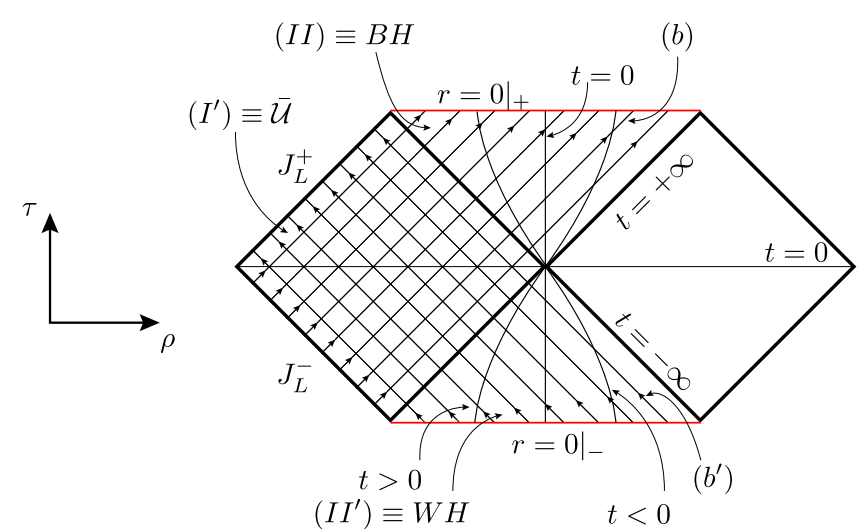

( $\beta)$

Figure 3. Penrose diagrams of radial null geodesics in SKS spacetime. $(\alpha)$ : $(a)$ : ingoing, $\left(a^{\prime}\right)$ : outgoing; $(\beta)$ : $(b)$ : ingoing, $\left(b^{\prime}\right)$ : outgoing

i) $\Theta$, the expansion scalar, given by the covariant divergence of the tangent vectors to the geodesics,

$$
\Theta=k_{; \mu}^{\mu},
$$

measures the fractional rate of change of the cross sectional area to the geodesics (i.e. how much the congruence diverges or converges if initially $\Theta>0$ or $\Theta<0$ );

ii) $\omega_{\mu \nu}=-\omega_{\nu \mu}$ is the rotation tensor;

iii) $\sigma_{\mu \nu}=\sigma_{\nu \mu}$ is the shear tensor;

and

iv) $R_{\mu \nu}$ is the Ricci tensor.

$\left(\omega_{\mu \nu}\right.$ and $\sigma_{\mu \nu}$ obey Raychaudhuri equations similar to (34).)

For a vacuum solution $R_{\mu \nu}=0$; also, for the Schwarzschild case, $\omega_{\mu \nu}=\sigma_{\mu \nu}=0$. So, the resulting Raychaudhuri equation is

$$
\frac{d \Theta}{d \lambda}=-\frac{1}{2} \Theta^{2}
$$

Using (17), (18), (23) and (16), a straightforward calculation leads to:

$$
\begin{gathered}
\Theta_{\text {in }}^{(a)}=\Theta_{i n}^{(b)}=-\frac{2}{r} \in(-\infty, 0), \\
\rightarrow-\infty, r \rightarrow 0_{+}(\text {future singularity }), \\
\rightarrow 0_{-}, r \rightarrow+\infty\left(\text { right } J_{R}^{-} \text {and left } J_{L}^{-} \text {past null infinities }\right), \\
\frac{d \Theta_{\text {in }}^{(x)}}{d(-r)}=-\frac{1}{2}\left(\Theta_{\text {in }}^{(x)}\right)^{2}, x=a, b ; \\
\Theta_{\text {out }}^{\left(a^{\prime}\right)}=\Theta_{\text {out }}^{\left(b^{\prime}\right)}=+\frac{2}{r} \in(0,+\infty), \\
\rightarrow+\infty, r \rightarrow 0_{+}(\text {past singularity }), \\
\frac{\left.d \Theta_{+}^{(y)}, r \rightarrow+\infty \text { right } J_{R}^{+} \text {and left } J_{L}^{+} \text {future null infinities }\right),}{d r}=-\frac{1}{2}\left(\Theta_{\text {out }}^{(y)}\right)^{2}, y=a^{\prime}, b^{\prime} .
\end{gathered}
$$

At the horizons $r=2 M$,

$$
\Theta_{\text {in }}^{(x)}=-\frac{1}{M}, \Theta_{\text {out }}^{(y)}=+\frac{1}{M} .
$$


(37) and (38) say that each point of the future singularity is a caustic of the $(a)$ and $(b)$ congruences. Obviously, the Raychaudhuri equation looses its validity at these points and at the past singularity. Classically, however, this fact is harmless since both $r=0$ future and past singularities do not belong to the spacetime. Instead, as will be shown in the next section, the eikonal functions are well behaved in the neighbourhood of these points.

\section{$5 \quad$ Eikonal Equations}

In the geometric optics approximation i.e. in the short wave length $l$ limit $(l<<M)$, light rays are represented by null geodesics whose tangent vectors $k^{\mu}$ are proportional to the wave number vectors $\tilde{k}^{\mu}$ given by the gradient of the eikonal function $\phi(t, r)$ :

$$
\tilde{k}_{\mu}=\frac{\partial \phi}{\partial x^{\mu}}
$$

with $[\phi]=[L]^{0}$. So, $\tilde{k}_{\mu}$ is normal to the surfaces $\phi=$ const. Since $\left[\tilde{k}_{\mu}\right]=[L]^{-1}$ and $\left[k_{\mu}\right]=[L]^{0}$, by dimensional reasons the relation between both vectors is

$$
k_{\mu}=M \tilde{k}_{\mu}
$$

since $M$ is the unique length scale of the problem. For the contravariant components one has

$$
k^{t}=g^{t t} M \tilde{k}_{t}=M f^{-1} \partial_{t} \phi, k^{r}=g^{r r} M \tilde{k}_{r}=-M f \partial_{r} \phi,
$$

and (24) and (25) become

$$
\frac{-2 M}{r^{2} f} \dot{\phi}+\dot{\phi}^{\prime} \mp \frac{2 M^{2}}{r^{2}} \dot{\phi} \phi^{\prime}=0
$$

and

$$
\frac{-2 M}{r^{2}} \phi^{\prime}-f \phi^{\prime \prime}=0
$$

where again the upper (lower) sign refers to outgoing (ingoing) rays, $\dot{\phi}=\frac{\partial \phi}{\partial t}, \phi^{\prime}=\frac{\partial \phi}{\partial r}$, $\dot{\phi}^{\prime}=\frac{\partial^{2} \phi}{\partial r \partial t}$, and $\phi^{\prime \prime}=\frac{\partial^{2} \phi}{\partial r^{2}}$. Equations (49) and (50) are the eikonal equations for $\phi=\phi_{\text {out }}$ (upper sign) and $\phi=\phi_{\text {in }}$ (lower sign).

It can be easily shown that for the $\left(a^{\prime}\right),(a),(b)$, and $\left(b^{\prime}\right)$ geodesics, the eikonal functions which reproduce the tangent vectors given by (19), (20), and $\left.k_{\text {out }}^{\mu}\right|_{\left(b^{\prime}\right)}$ and $\left.k_{i n}^{\mu}\right|_{(b)}$ in (23), and obey the equations (49) and (50), are respectively given by

$$
\begin{gathered}
\phi_{\text {out }}^{(a \prime)}(t, r)=\frac{t}{M}-\frac{r}{M}-2 \ln \left|\frac{r}{2 M}-1\right|=\frac{u(t, r)}{M}, \\
\phi_{\text {in }}^{(a)}(t, r)=\frac{t}{M}+\frac{r}{M}+2 \ln \left|\frac{r}{2 M}-1\right|=\frac{v(t, r)}{M}, \\
\phi_{\text {out }}^{(b \prime)}(t, r)=-\frac{t}{M}-\frac{r}{M}-2 \ln \left|\frac{r}{2 M}-1\right|,
\end{gathered}
$$

and

$$
\phi_{i n}^{(b)}(t, r)=-\frac{t}{M}+\frac{r}{M}+2 \ln \left|\frac{r}{2 M}-1\right| .
$$

The values that these eikonal functions take at the past and future: singularities, horizons, and null infinities, are given in the following tables:

For all geodesic congruences, the $t$ values can be read from the Penrose (or Kruskal) diagram as the $t$-line passing through the intersection of the light ray in question with the past or future singularity. Except for light travelling along the future $\left(H_{+}\right)$or past $\left(H_{-}\right)$horizons, where $t= \pm \infty$, the corresponding eikonal is finite at the singularities. Also, the fact that $\phi_{\text {out }}^{\left(a^{\prime}\right),(b \prime)}\left(t, 0_{+}\right), \phi_{\text {in }}^{(a),(b)}\left(t, 0_{+}\right) \sim \frac{1}{M}$, suggests that 
Table 1. Eikonal values at null infinities, horizons and singularities of the $(a)$ and $\left(a^{\prime}\right)$ geodesic congruences

\begin{tabular}{lllllll}
\hline & $J_{R}^{-}$ & $J_{R}^{+}$ & $H_{+}$ & $H_{-}$ & $r=\left.0\right|_{-}$ & $r=\left.0\right|_{+}$ \\
\hline$\phi_{\text {out }}^{\left(a^{\prime}\right)}$ & - & $-\infty$ & - & $+\infty$ & $t / M$ & $t$ \\
$\phi_{\text {in }}^{\left(a^{\prime}\right)}$ & $+\infty$ & - & $-\infty$ & - & & $t / M$ \\
\hline
\end{tabular}

Table 2. Eikonal values at null infinities, horizons and singularities of the $(b)$ and $\left(b^{\prime}\right)$ geodesic congruences

\begin{tabular}{lllllll}
\hline & $J_{L}^{-}$ & $J_{L}^{+}$ & $H_{+}$ & $H_{-}$ & $r=\left.0\right|_{-}$ & $r=\left.0\right|_{+}$ \\
\hline$\phi_{\text {out }}^{\left(b^{\prime}\right)}$ & - & $-\infty$ & $+\infty$ & - & $-t / M$ & - \\
$\phi_{\text {in }}^{\left(b^{\prime}\right)}$ & $+\infty$ & - & - & $-\infty$ & - & $-t / M$ \\
\hline
\end{tabular}

these quantities are proportional to the Hawking temperature $\frac{1}{8 \pi M}$. In fact, through the formal association of the mechanical action

$$
S=\hbar M \phi
$$

and reinserting units, one obtains

$$
S_{\text {out }}^{\left(a^{\prime}\right)}\left(t, 0_{+}\right)=S_{\text {in }}^{(a)}\left(t, 0_{+}\right)=8 \pi k_{B} T_{H} t, S_{\text {out }}^{\left(b^{\prime}\right)}\left(t, 0_{+}\right)=S_{\text {in }}^{(b)}\left(t, 0_{+}\right)=-8 \pi k_{B} T_{H} t
$$

with $k_{B}$ the Boltzmann constant and $T_{H}=\frac{\hbar c^{3}}{8 \pi k_{B} G M}$ the Hawking temperature.

\section{Discussion}

We study the eikonal function $\phi$ associated with the short wave lenght limit $(l<<M)$ of radial light rays propagating in the ideal eternal Schwarzschild-Kruskal-Szekeres-Penrose black/white hole. (Applications of the eikonal concept in general relativity and the equivalent geometric optics approximation in black holes, are reviewed in references [11] and [12].) These rays are represented by null geodesics and there are two sources of them: the past singularity $r=\left.0\right|_{-}$, where the outgoing geodesic congruences end in the right and left future null infinities $J_{R}^{+}$and $J_{L}^{+}$, and the left and right past null infinities $J_{L}^{-}$and $J_{R}^{-}$, where the ingoing geodesics end in the future singularity $r=\left.0\right|_{+} \cdot \phi$ turns out to be finite in the neighbourhood of the singularities, proportional to $1 / M$, the inverse mass of the hole. Instead, $\phi$ diverges at the horizons $r=2 M$. In contradistinction, and for comparison, the expansion scalar $\Theta$ of the Raychaudhuri equation obeyed by the congruences has the opposite behavior: it is finite at the horizons and proportional to $1 / M$ but diverges at the singularities: $\theta_{\text {in }} \rightarrow-\infty$ at $r=\left.0\right|_{+}$and $\Theta_{\text {out }} \rightarrow+\infty$ at $r=\left.0\right|_{-}$. Though the whole discussion is classical, it is nevertheless of interest to study the behavior of quantities like the eikonal (related to the wave propagation of light and its quantization) and the expansion (related to the attractive nature of gravity) in the interior of the black holes. It is clear that a full understanding of what happens at the singularities has to wait for a theory of quantum gravity.

Acknowledgments. The author thanks Oscar Brauer for the realization of the Figures.

\section{References}

1. Blandford, R.D. and Thorne, K.S. Applications of Classical Physics, Chapter 7, Caltech, (2013).

2. Landau, L.D. and E. M. Lifshitz, The Classical Theory of Fields, Course of Theoretical Physics Vol. 2, Elsevier, (1975).

3. Kruskal, M.D., Maximal extension of Schwarzschild metric, Phys. Rev. 119, 1743-1745, (1960).

4. Szekeres, G. On the singularities of a Riemannian manifold, Publ. Math. Debrecen 7, 285-301, (1960).

5. Kar. S. and Sengupta, S. The Raychaudhuri equations: A brief review, Pramana 69, 49-76, (2007).

6. Baez, J.C. and Bunn, E.F. The meaning of Einstein's equation, Am. J. Phys. 73, 644-652, (2005). 
7. Poisson, E. A Relativist's Toolkit, The Mathematics of Black Hole Mechanics, Cambridge University Press, (2004).

8. Finkelstein, D. Past-future asymmetry of the gravitational field of a point particle, Phys. Rev. 110, 965-967, (1958).

9. Penrose, R. The Light Cone at Infinity. In Proceedings of the 1962 Conference on Relativistic Theories of Gravitation, Warsaw; Polish Academy of Sciences, (1965).

10. Carter, B. Complete analytic extension of the symmetry axis of Kerr's solution of Einstein's equations, Phys. Rev. 141, 1242-1247, (1966).

11. Ni, W.-T. "Equivalence principles, spacetime structure and the cosmic connection" in One Hundred Years of General Relativity, Vol 1, Chapter 5, World Scientific, (2017).

12. Frolov, V.P. and Zelnikov, A.Z. "Introduction to Black Hole Physics", Oxford University Press, (2015). 